To the editor: We read with interest your September 2010 Vol 12, No 5 cover article "Hookworm-Related Cutaneous Larva Migrans." ${ }^{1}$ In it, the American authors state that one of three drugs should be used to eradicate the larvae: ivermectin, albendazole, or thiabendazole.

As we found out recently with two cases of cutaneous larva migrans, these drugs are not available in any Canadian pharmacy. Health Canada provides these drugs but requires the physician and local pharmacist to complete a Special Access Program Form $^{2}$ and fax it to them in Ottawa. The turnaround time with release and shipment of the drug can take several days, but quicker release can be obtained by contacting the Therapeutics Drug Directorate office through its 24-hour emergency hotline at 613-941-2108.

A second-line drug, mebendazole (Vermox), is more readily available., In both of our cases, however, high doses of mebendazole over several days (200 mg orally twice daily for 3 days) failed to produce permanent relief of symptoms. Only with one of the three listed drugs of choice, albendazole, were the patients cured.

It seems that diagnosing these exotic rashes in Canada is only half of the battle; getting the appropriate medications to the patient remains the other half.

\section{Brian Steinhart, MD}

Emergency Physician, St. Michael's Hospital, Department of Emergency Services, Toronto, ON

\section{Ann Kosinski}

Research Pharmacy Distribution Assistant, Research Pharmacy, St. Michael's Hospital, Toronto, ON

\section{References}

1. Tomich EB, Knutson T, Welsh L. Hookworm-related cutaneous larva migrans. CFEM 2010;12:446.

2. Available at: http://www.hc-sc.gc.ca/dhpmps/acces/drugs-drogues/sapg3_pasg3eng.php.

3. Caumes E. Treatment of cutaneous larva migrans and Toxocara infection. Fundam Clin Pharmacol 2003;17: 213.

4. Bouchaud O, Houze S, Schiemann R, et al. Cutaneous larva migrans in travelers: a prospective study, with assessment of therapy with ivermectin. Clin Infect Dis 2000;31:493. 